\title{
Antimicrobial therapy for acute cholangitis: Tokyo Guidelines
}

\author{
Atsushi Tanaka ${ }^{1}$, Tadahiro Takada ${ }^{2}$, Yoshifumi Kawarada ${ }^{3}$, Yuji Nimura ${ }^{4}$, Masahiro Yoshida ${ }^{2}$, \\ Fumihiko Miura ${ }^{2}$, Masahiko Hirota ${ }^{5}$, Keita Wada 2 , Toshihiko Mayumi ${ }^{6}$, Harumi Gomi ${ }^{7}$, \\ Joseph S. Solomkin ${ }^{8}$, Steven M. Strasberg ${ }^{9}$, Henry A. Pitt ${ }^{10}$, Jacques Belghiti ${ }^{11}$, Eduardo de Santibanes ${ }^{12}$, \\ Robert Padbury ${ }^{13}$, Mirn-Fu Chen ${ }^{14}$, Giulio Belli ${ }^{15}$, Chen-Guo Ker ${ }^{16}$, Serafin C. Hilvano ${ }^{17}$, Sheung-Tat Fan ${ }^{18}$, \\ and Kui-Hin Liau ${ }^{19}$

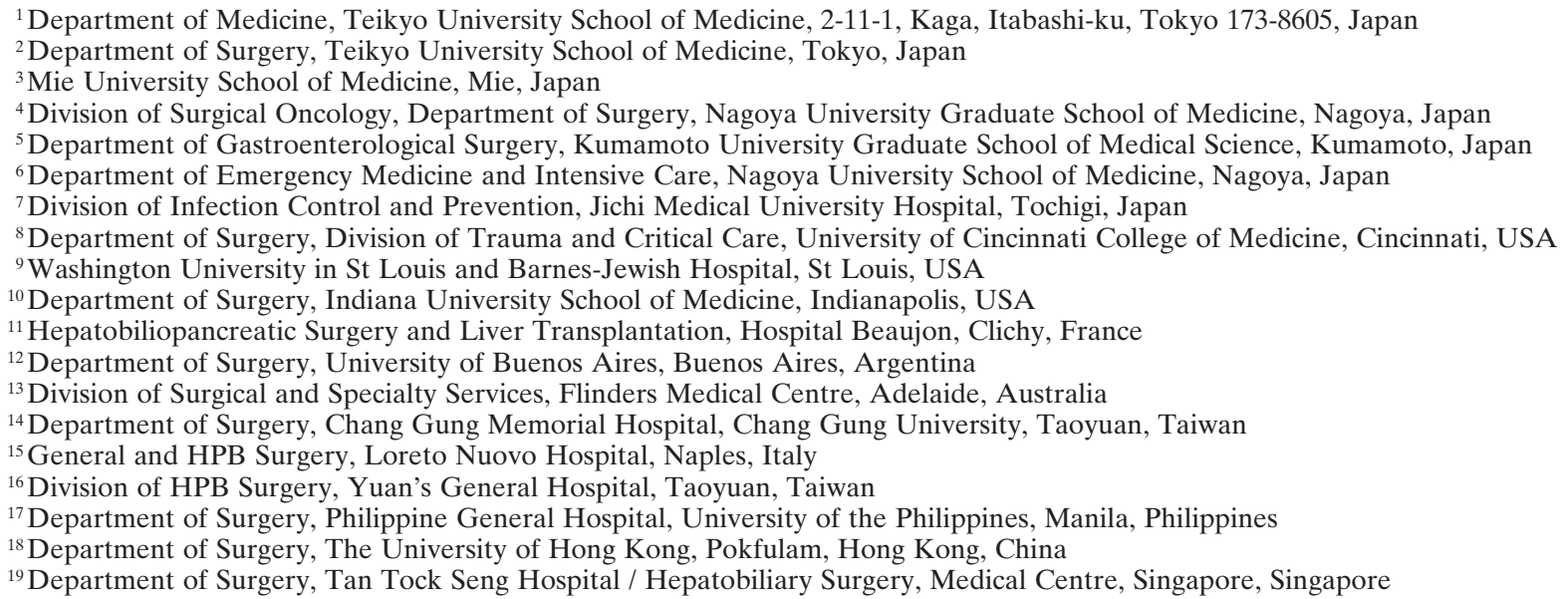

\begin{abstract}
Antimicrobial agents should be administered to all patients with suspected acute cholangitis as a priority as soon as possible. Bile cultures should be performed at the earliest opportunity. The important factors which should be considered in selecting antimicrobial therapy include the agent's activity against potentially infecting bacteria, the severity of the cholangitis, the presence or absence of renal and hepatic diseases, the patient's recent history of antimicrobial therapy, and any recent culture results, if available. Biliary penetration of the microbial agents should also be considered in the selection of antimicrobials, but activity against the infecting isolates is of greatest importance. If the causative organisms are identified, empirically chosen antimicrobial drugs should be replaced by narrower-spectrum antimicrobial agents, the most appropriate for the species and the site of the infection.
\end{abstract}

Key words Cholangitis · Anti-infective agents · Guidelines · Infection · Biliary

Offprint requests to: A. Tanaka

Received: May 31, 2006 / Accepted: August 6, 2006

\section{Introduction}

In the medical treatment, of acute cholangitis, antimicrobial agents should be chosen empirically and carefully. As soon as a diagnosis of acute cholangitis is considered, antimicrobial agents should be selected empirically, with careful consideration of several factors, including antimicrobial activity against the causative bacteria, the severity of the cholangitis, the presence/ absence of renal and hepatic disease, a recent (1-year) history of antimicrobial therapy, local susceptibility patterns (antibiogram), and (although controversies still exist) the biliary penetration of the antimicrobial agents. Whenever any presumptive or empirical antimicrobial agents are used, they should be switched for the best available narrower-spectrum agents to avoid superinfection or the emergence of antimicrobial resistance as a cause of treatment failure. Long-term administration without an acceptable rationale should be avoided. In this article, we review previous bacteriological studies and clinical trials. We also provide current recommendations for the antimicrobial agents to be used for acute cholangitis, in an evidence- and consensus-based manner, on the basis of discussions at the Tokyo International Consensus Meeting. 
Table 1. Bacterial culture positive rates in bile (\%) in various biliary diseases

\begin{tabular}{|c|c|c|c|c|c|c|}
\hline & Bile & $\begin{array}{l}\text { Non-biliary } \\
\text { disease }\end{array}$ & $\begin{array}{l}\text { Chole- } \\
\text { lithiasis }\end{array}$ & $\begin{array}{c}\text { Acute } \\
\text { cholecystitis }\end{array}$ & $\begin{array}{c}\text { Choledo- } \\
\text { cholithiasis } \\
\text { (+cholangitis) }\end{array}$ & $\begin{array}{c}\text { Hepatolithiasis } \\
\text { (+cholangitis) }\end{array}$ \\
\hline Chang $(2002)^{4}$ & $\begin{array}{l}\text { Gallbladder } \\
\text { Bile duct }\end{array}$ & & 17.0 & 47.0 & 63.0 & 70.0 \\
\hline Csendes $(1996)^{5,6}$ & $\begin{array}{l}\text { Gallbladder } \\
\text { Bile duct }\end{array}$ & 0 & $\begin{array}{l}22.2 \\
23.9\end{array}$ & $\begin{array}{l}46.1 \\
29.0\end{array}$ & 58.2 & 93.9 \\
\hline Csendes $(1994)^{39}$ & Gallbladder & 0 & 32.0 & 41.0 & 58.0 & \\
\hline Maluenda $(1989)^{2}$ & $\begin{array}{l}\text { Bile duct } \\
\text { Gallbladder }\end{array}$ & 0 & & 43.0 (Chronic; 30) & 76.0 & 89.0 \\
\hline $\begin{array}{l}\text { Csendes }(1975)^{40} \\
\text { Kune }(1974)^{41}\end{array}$ & $\begin{array}{l}\text { Gallbladder wall } \\
\text { Gallbladder } \\
\text { Bile duct }\end{array}$ & 0 & 13.0 & $\begin{array}{l}47.0 \text { (Chronic; 33) } \\
54.0\end{array}$ & 59.0 & \\
\hline
\end{tabular}

Table 2. Bacterial species identified in bile of patients with acute cholangitis ${ }^{2,4-8}$

\begin{tabular}{lc}
\hline Bacteria & Positive rate in bile (\%) \\
\hline Aerobes & \\
Escherichia coli & $31-44$ \\
Klebsiella & $8.5-20$ \\
Enterobacter & $5-9.1$ \\
Proteus & $1-4.8$ \\
Salmonella typhi & $0.8-2.6$ \\
Salmonella paratyphi & $0.8-2.3$ \\
Citrobacter & $1.6-4.5$ \\
Pseudomonas & $0.5-7$ \\
Streptococcus spp. & $2-10$ \\
Enterococcus faecalis & $2.6-10$ \\
Anaerobes & \\
Clostridium & $3-12.7$ \\
Bacteroides & $0.5-8$ \\
\hline
\end{tabular}

\section{Q1. How to detect causative organisms of acute cholangitis?}

Bile/blood culture should be performed at all available opportunities (recommendation B).

Table 1 lists the positive rates of bacterial cultures in bile in various biliary diseases. While bile is sterile in individuals without any biliary disease, a positive bile culture is common in various biliary diseases. In patients with acute cholangitis and choledocholithiasis, a positive bile culture is correlated with progression to severe cholangitis and a high mortality rate (level 2b-3b). ${ }^{1,2}$ Also, care should be exercised regarding the postoperative occurrence of infective complications in patients with positive bile cultures (level 5). ${ }^{3}$ These facts emphasize the importance of early antimicrobial therapy.

It was reported that microbial organisms contained in bile from various biliary diseases were of intestinal bacterial flora origin (Table 2). Aerobic bacteria such as
Escherichia coli, Klebsiella, Enterococcus, and Enterobacter are most frequently isolated, whereas Streptococcus spp., Pseudomonas, and Proteus are less frequently isolated (level 2b-3b).,2,8 Although anaerobic bacteria such as Clostridium and Bacteroides are often isolated, most of these patients have polymicrobial infections with aerobic bacteria (level 5). ${ }^{9-11}$ There are reports that anaerobic bacteria are often detected patients with severe acute cholangitis (level 2b-3b). ${ }^{12-14}$

Moreover, it should also be kept in mind for the estimation of causative bacteria in acute cholangitis, whether the infection is community-acquired or hospital-acquired. When it is community-acquired, intestinal microorganisms such as E. coli, Klebsiella, and Enterococcus are likely to be the causative bacteria. By contrast, we have to take into account that, in patients with hospital-acquired type infections, especially those in a postoperative state or those with indwelling stents and malignancies, more resistant organisms, i.e., methicillinresistant Staphylococcus aureus (MRSA), vancomycinresistant enterococcus (VRE), and Pseudomonas, are frequently detected as causative microorganisms.

Many patients with cholangitis with a microbialpositive blood culture have the same species of bacteria in blood as those isolated from bile cultures (level 3b), ${ }^{12}$ and the positive rate increases with the co-existence of acute cholangitis due to biliary obstruction (level 2b). ${ }^{1}$ The blood culture-positive rates in acute cholangitis have been reported to vary from $21 \%$ to $71 \%$ (level 5). ${ }^{9-11,15}$ Patients with bacteremia are frequently resistant to treatment regimens (level 4), ${ }^{16}$ and bacteremia is correlated with the duration of hospitalization, the incidence of postoperative renal failure, and the mortality rate (level $2 b$ ). ${ }^{1}$ These findings underscore the importance of antisepsis therapy, as outlined in the Surviving Sepsis Campaign guidelines of the Society of Critical Care Medicine. ${ }^{17}$

There has been no good-quality evidence to support the importance of blood and bile culture in patients with 


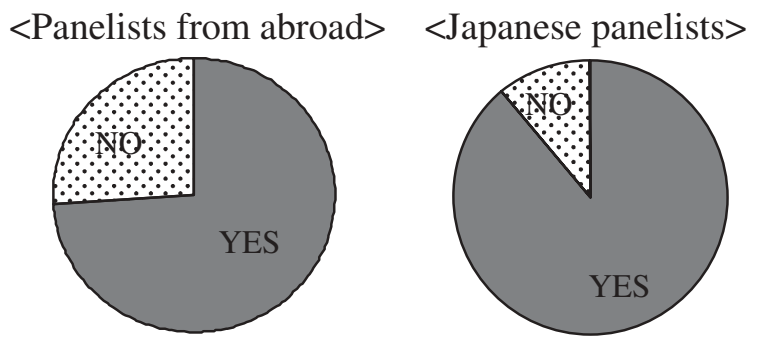

Fig. 1. Responses to the question: "Should bile culture be performed in all patients with acute cholangitis?" Yes, 26 (74\%); no, 9 (26\%) in 35 overseas panelists, and yes, 17 (89\%); no, $2(11 \%)$ in 19 Japanese panelists

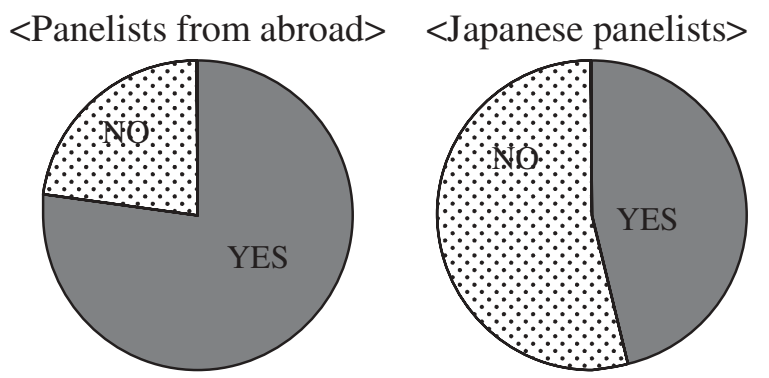

Fig. 2. Responses to the question: "Should blood culture be performed in all patients with acute cholangitis?" Yes, 20 (77\%); no, 6 (23\%) in 26 overseas panelists, and yes, 12 (46\%); no, $14(54 \%)$ in 26 Japanese panelists

acute cholangitis. At the Tokyo Consensus Meeting, we reached a consensus on the importance of bile culture for patients with acute cholangitis (Fig. 1). By contrast, there was a significant discrepancy between Japanese and overseas panelists in regard to the importance placed on blood culture for all patients; while more than half of the overseas panelists agreed on the necessity for blood culture, most of the Japanese panelists disagreed (Fig. 2). Representative reasons for the disagreement were that, usually, blood cultures did not provide any information beyond that provided by bile cultures, and that postoperative acute cholangitis in patients with a choledocho-jejuno anastomosis did not need intensive bacteriological studies. It is, however, rational to rule out bacteremia, when possible, in patients with severe cholangitis, as this would affect the duration of antimicrobial therapy.

Q2. How are antimicrobial agents used for patients with acute cholangitis?

- Antimicrobial agents should be administered to all patients diagnosed as having acute cholangitis (recommendation A); the Antimicrobial agents should be administered as soon as the diagnosis of acute cholangitis is suspected or established.
- For patients with moderate (grade II) or severe (grade III) acute cholangitis, antimicrobial agents should be administered for a minimum duration of 5-7 days. More prolonged therapy could be required, depending on the presence of bacteremia and the patient's clinical response, judged by fever, white blood cell count, and $\mathrm{C}$ reactive protein, when available (recommendation A).

- For patients with mild (grade I) acute cholangitis, the duration of antimicrobial therapy could be shorter ( 2 or 3 days) (recommendation $A$ ).

An important and fruitful discussion was held regarding the duration of antimicrobial therapy for patients with acute cholangitis (see "Discussion"). In summary, patients with moderate (grade II) or severe (grade III) acute cholangitis should receive a minimum duration of therapy of 5-7 days, and then, based on the anatomy of the disease and the presence of bacteremia, and their clinical responses, patients may need more prolonged therapy. However, for the large group of patients with mild (grade I) cholangitis, 2 or 3 days of antimicrobial therapy is likely to be sufficient. Needlessly prolonged antimicrobial therapy risks adverse reactions to the antimicrobials, and intensifies pressure for the development and acquisition of resistant bacteria.

Q3. What are the most important factors for consideration in antimicrobial drug selection?

(1) Antimicrobial activity against causative bacteria

(2) Severity of cholangitis

(3) Presence/absence of renal and hepatic disease

(4) Past history of antimicrobial administration to the patient

(5) Local susceptibility patterns (antibiogram) of the suspected causative organisms

(6) Biliary penetration of the antimicrobial agents.

The dose of the antimicrobial agent should be reduced for patients with reduced renal function. Because most cephalosporin, penicillin, aminoglycoside, and carbapenem antimicrobial drugs are excreted by the kidneys, the dose is reduced for patients with nephropathy and decreased renal function. The Sanford guide to antimicrobial therapy, $2003^{18}$ and Goodman and Gilman's the pharmacological basis of therapeutics ${ }^{19}$ recommend that renal function be estimated by the following formula:

Creatinine clearance predicted from serum creatinine $(\times 0.85$ for females $)=(140-$ age $)($ optimum body weight $(\mathrm{kg})) /(72 \times$ serum creatinine $\mathrm{mg} / \mathrm{dl})$ 
where male optimum body weight is $50.0 \mathrm{~kg}+0.91 \mathrm{~kg} /$ $\mathrm{cm}(150 \mathrm{~cm}$ and taller) and female optimum body weight is $45.5 \mathrm{~kg}+0.91 \mathrm{~kg} / \mathrm{cm}(150 \mathrm{~cm}$ and taller $)$.

\section{Drug dosage adjustment should be done in pa- tients with decreased renal function. The Sanford guide to antimicrobial therapy and Goodman and Gilman's the pharmacological basis of therapeu- tics should be consulted (recommendation A).}

Drug dosage adjustment for ceftriaxone is not necessary in patients with renal failure. But dose adjustment of ceftriaxone is indicated for patients with severe hepatic impairment. ${ }^{18}$ In addition, when biliary obstruction that blocks the enterohepatic circulation of bile is present, the administration of third- and fourth-generation cephalosporins may replace the intestinal flora and disturb vitamin $\mathrm{K}$ absorption, in turn risking coagulopathic hemorrhage. This phenomenon, leading to bleeding tendency, can be enhanced in patients with comorbid liver diseases or liver failure due to severe acute cholangitis. Intravenous administration of vitamin $\mathrm{K}$ may be indicated in these situations.

\section{Q4. Should biliary penetration be considered} important in the selection of therapeutic antimicrobials in acute cholangitis?

Biliary penetration should be considered in the selection of antimicrobial agents in acute cholangitis (recommendation A).
It has been debated whether antimicrobials with good biliary penetration should be recommended for acute cholangitis. Indeed, there was a common belief, particularly in Japan, that antimicrobial agents with excellent biliary penetration are more effective for the treatment of acute cholangitis. However, there are no clinical or experimental data to strongly support the recommendation of antimicrobials with excellent biliary penetration for these patients. In fact, in most patients with acute cholangitis, biliary obstruction is usually present, and antimicrobial drugs may not be detected in bile even if they demonstrate excellent biliary excretion in normal conditions (level 3b-4). ${ }^{20-27}$

Nevertheless, at the Consensus Meeting, we reached a consensus that the importance of biliary penetration should be emphasized for the empirical selection of antimicrobial agents (Fig. 3). For details, see "Discussion at the Tokyo International Consensus Meeting." In Table 3, we list antimicrobial agents with good biliary penetration.

\section{Q5. What are the results of clinical trials regarding antimicrobial therapy in acute cholangitis?}

The combination of ampicillin and an aminoglycoside was regarded as a standard regimen for cholangitis in the 1980s (level 4-5), ${ }^{28,29}$ and most randomized controlled trials (RCTs) have concluded that recently developed antimicrobial drugs had effectiveness and usefulness equivalent to that of ampicillin and aminoglycosides (Table 4) (level 2b). ${ }^{30-35}$ Therefore, according
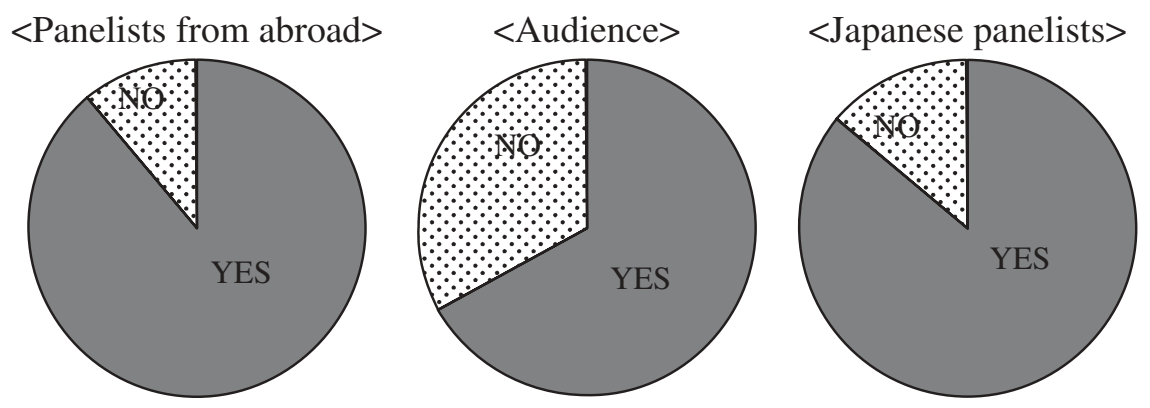

Fig. 3. Responses the question: "Should the biliary penetration of antimicrobial agents be considered important in the in selection in moderate (grade II) or severe (grade III) acute cholangitis?" Yes, 24 $(89 \%)$; no, $3(11 \%)$ in 27 overseas panelists; yes, 18 (67\%); no, 9 (33\%) in 27 Japanese panelists; and yes, 55 (86\%); no, 9 $(14 \%)$ in 64 audience members

Table 3. Intravenous antimicrobial drugs with good biliary penetration (level 4) ${ }^{18}$

\begin{tabular}{ll}
\hline Penicillins & \multicolumn{1}{c}{ Piperacillin, aspoxicillin, piperacillin/tazobactam, ampicillin } \\
\hline $\begin{array}{l}\text { Cephalosporins } \\
\text { 1st Generation }\end{array}$ & Cefazoline \\
2nd Generation & Cefmetazole, cefotiam, flomoxef \\
3rd, 4th Generation & Cefoperazone/sulbactam, ${ }^{20}$ ceftriaxone, ${ }^{42}$ cefozopran, cefpirome, ceftazidime, cefoperazone \\
Fluoroquinolones & Ciprofloxacin, ${ }^{20}$ Pazufloxacin \\
Monobactams & Aztreonam ${ }^{21}$ \\
Lincosamides & Clindamycin \\
\end{tabular}


Table 4. Comparative tests clinical of antimicrobial drugs in acute cholangitis

\begin{tabular}{|c|c|c|c|c|}
\hline Authors (Year) & Subjects & Administered antimicrobials & Clinical cure rate & $\begin{array}{c}\text { Statistical } \\
\text { significance }\end{array}$ \\
\hline \multirow[t]{3}{*}{ Muller $(1987)^{30}$} & \multirow[t]{3}{*}{ Cholangitis } & Ampicillin+ tobramycin & $85 \%(17 / 20)$ & \\
\hline & & Piperacillin & $60 \%(9 / 15)$ & NS \\
\hline & & Cefoperazone & $56 \%(10 / 18)$ & $P<0.05$ \\
\hline \multirow[t]{2}{*}{ Gerecht $(1989)^{31}$} & \multirow[t]{2}{*}{ Cholangitis } & Mezocillin & $83 \%(20 / 24)$ & $P<0.01$ \\
\hline & & Ampicillin + gentamicin & $41 \%(9 / 22)$ & \\
\hline \multirow[t]{2}{*}{ Thompson $(1990)^{32}$} & \multirow[t]{2}{*}{ Cholangitis } & Piperacillin & $70 \%$ & NS \\
\hline & & Ampicillin + tobramycin & $69 \%$ & \\
\hline \multirow[t]{2}{*}{ Chacon $(1990)^{33}$} & \multirow[t]{2}{*}{ Cholangitis + cholecystitis } & Pefloxacin & $98 \%(49 / 50)$ & NS \\
\hline & & Ampicillin + gentamicin & $95.7 \%(45 / 47)$ & \\
\hline \multirow[t]{2}{*}{ Thompson $(1993)^{34}$} & \multirow[t]{2}{*}{ Cholangitis + cholecystitis } & Cefepime & $97.5 \%(78 / 80)$ & NS \\
\hline & & Mezlocillin + gentamicin & $100 \%(40 / 40)$ & \multirow{3}{*}{ NS } \\
\hline \multirow[t]{2}{*}{ Sung $(1995)^{35}$} & \multirow[t]{2}{*}{ Cholangitis } & Ciprofloxacin & $85 \%(39 / 46)$ & \\
\hline & & Ceftazidime + ampicillin + metronidazole & $77 \%(34 / 44)$ & \\
\hline
\end{tabular}
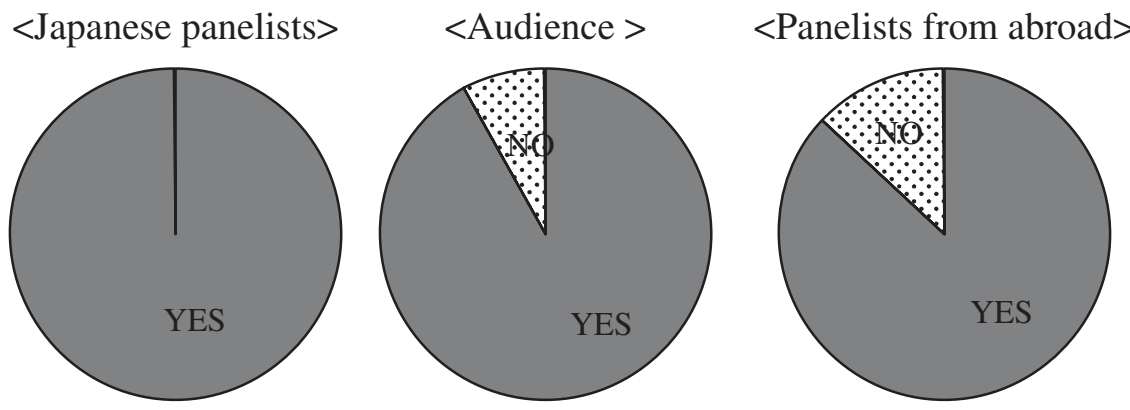

Fig. 4. Reponses to the question: "Should empirically administered antimicrobial drugs be changed for more appropriate agents, according to the identified causative microorganisms and their sensitivity to antimicrobials?" Yes, $30(100 \%)$ in 30 Japanese panelists; yes, 21 (87\%); no, 3 $(13 \%)$ in 24 panelists from abroad; and yes, 61 (92\%); no, $5(8 \%)$ in 66 audience members

to the clinical trials available so far, piperacillin, ampicillin and an aminoglycoside, and several cephalosporins, are recommended for the treatment of acute cholangitis.

However, at present antimicrobial agents widely used for acute cholangitis, including penicillin/ $\beta$-lactamase inhibitors, carbapenems, and the third- and fourthgeneration cephalosporins, have not been tested in these RCTs. In this regard, we recommend the alternative regimens for antimicrobial agents stated in the Tokyo Guidelines. The recommendations were reached in a consensus-based manner, as follows.

Q6. What are the current recommendations for antimicrobial therapy in acute cholangitis?

- Antimicrobial drugs should be selected according to the severity assessment (recommendation A).

- Empirically administered antimicrobial agents should be changed for more appropriate agents according to the identified causative microorganisms and their sensitivity to antimicrobials (recommendation $\mathbf{A}$ ).
In the Infectious Diseases Society of America (IDSA) guidelines for intraabdominal infections, the selection of antimicrobial agents is based on the severity of the infection. ${ }^{36}$ In the Tokyo Guidelines, the selection of antimicrobial agents is based on the severity of acute cholangitis. However, it should be emphasized that there is little high-level evidence that supports this notion.

It was widely accepted at the Consensus Meeting that empirically administered antimicrobial agents should be changed for more appropriate agents according to the identified causative microorganisms and their sensitivity to antimicrobials (Fig. 4).

In any guidelines, recommended doses of antimicrobials, ideally based on body weight, should also be provided. However, the dose administered can vary in each country, depending on medical practices and legal regulations. For instance, it was known and discussed at the Consensus Meeting that the legally approved doses of antimicrobials in Japan are different from those used in the United States and Europe. Therefore, recommended doses of antimicrobial agents are not provided in the Tokyo Guidelines, and doses should be determined according to local rules and regulations. Similarly, the cost of the agents, which should also be discussed, varies in 
different countries and was not addressed in the Tokyo Guidelines.

Antibacterials selected for the three grades of acute cholangitis

Mild (grade I) acute cholangitis

Mild (grade I) cases of the disease are often caused by a single intestinal organism, such as E. coli, and therefore monotherapy with one of the following antimicrobial drugs should be chosen. Because intestinal organisms producing $\beta$-lactamase, which are resistant to penicillins and cefazoline, are likely to be detected, the use of a penicillin/ $\beta$-lactamase inhibitor, such as piperacillin/ tazobactam, ${ }^{37}$ or ampicillin/sulbactam is recommended (see Table 5).

Moderate (grade II) and severe (grade III) acute cholangitis (Table 6)

Patients with moderate (grade II) and severe (grade III) disease are often infected with multiple and/or resistant organisms (level 2b-3b).,32,14 Thus, third- and fourthgeneration cephalosporins, with a wide antimicrobial spectrum, as well as broadspectrum penicillin/ $\beta$-lactamase inhibitors, are recommended as the drug of first choice. Depending on the local susceptibility patterns (antibiogram), if the drug of first choice is ineffective, fluoroquinolones and carbapenems can be used.

It should be emphasized that the inappropriate use or overuse of third- and fourth-generation cephalosporins and carbapenems would likely result in the emergence of resistant bacteria. For instance, it has been reported that some $E$. coli strains acquire resistance to ampicillin/sulbactam.

Piperacillin/tazobactam is strongly recommended when Pseudomonas spp. are considered as the causative

Table 5. Antibacterials for grade I acute cholangitis

\begin{tabular}{ll}
\hline $\begin{array}{l}\text { First-generation cephalosporins } \\
\text { Second-generation }\end{array}$ & Cefazoline \\
cephalosporins & Cefmetazole, cefotiam, \\
Penicillin//3-lactamase inhibitor & oxacephem, flomoxef
\end{tabular}

organisms. Of note, the ratio of penicillin to tazobactam is different in Japan $(4: 1)$ from that in the United States $(8: 1)$.

\section{Q7. Is there any difference between Japan and the United States in the use of antimicrobial agents for acute cholangitis?}

On the basis of pharmacokinetics and pharmacodynamics, there is a significant difference between the United States and Japan in antimicrobial dosing regimens. For details, see "Discussion", for discussions held at the Tokyo International Consensus Meeting.

As a consequences of the inappropriate dosing regimens in Japan, inadequate clinical responses may be seen in Japanese patients. Moreover, the overuse of broad spectrum agents such as carbapenems has been another problem in Japan. Unpublished data from a major global pharmaceutical company indicate that Japan consumes half of the carbapenems produced worldwide. This could be evidence of the overuse of carbapenems in Japan.

\section{Q8. How should antimicrobial drugs be administered for acute cholangitis associated with biliary obstruction?}

The presence of biliary obstruction may significantly influence the biliary penetration of the antimicrobial, as well as acting as a persistent source of infection. Therefore, patients with acute cholangitis, especially those with severe (grade III) disease, should have immediate biliary drainage along with appropriate antimicrobial therapy (recommendation A).

When biliary obstruction is present, even an antimicrobial drug with excellent biliary excretion may not enter the bile tract (level 3b-4) ${ }^{20-27}$ The active transfer of antimicrobial drugs into bile is not restored early after the biliary obstruction has been relieved (level 4). ${ }^{25,38}$ Therefore, immediate biliary drainage, as well as the admin-

Table 6. Antibacterials for moderate (grade II) and severe (grade III) acute cholangitis

First options

Wide spectrum penicillin/ $\beta$-lactamase inhibitor (as single agents)

Third- and fourth-generation cephalosporins

Monobactams

One of above + metronidazole (to cover anaerobes)

Second options

Fluoroquinolones

One of above + metronidazole (to cover anaerobes)

Carbapenems
Ampicillin/sulbactam, piperacillin/tazobactam

Cefoperazone/sulbactam, ceftriaxone, ceftazidime, cefepime, cefozopran

Aztreonam

Ciprofloxacin, levofloxacin, pazufloxacin

Meropenem, imipenem/cilastatin, doripenem 
istration of antimicrobials, is crucial in view of controlling the source of infection.

Acknowledgments. We would like to express our deep gratitude to the Japanese Society for Abdominal Emergency Medicine, the Japan Biliary Association, and the Japanese Society of Hepato-Biliary-Pancreatic Surgery, who provided us with great support and guidance as part of the Preparation of the Guidelines. This process was conducted as part of the Project on the Preparation and Diffusion of Guidelines for the Management of Acute Cholangitis (H-15-Medicine-30), with a research subsidy for fiscal 2003 and 2004 (Integrated Research Project for Assessing Medical Technology) sponsored by the Japanese Ministry of Health, Labour, and Welfare.

We also truly appreciate the panelists who cooperated with and contributed significantly to the International Consensus Meeting, held in Tokyo on April 1 and 2, 2006 .

\section{References}

1. Pitt HA, Postier R, Cameron J. Consequences of preoperative cholangitis and its treatment on the outcome of operation for choledocholithiasis. Surgery 1983;94:447-52. (level 2b)

2. Maluenda F, Csendes A, Burdiles P, Diaz J. Bacteriological study of choledochal bile in patients with common bile duct stones, with or without acute suppurative cholangitis. Hepatogastroenterology 1989;36:132-5. (level 3b)

3. Claesson B. Microflora of the biliary tree and liver - clinical correlates. Dig Dis 1986;4:93-118. (level 5)

4. Chang W, Lee K, Wang S, Chuang S, Kuo K, Chen J, et al. Bacteriology and antimicrobial susceptibility in biliary tract disease: an audit of 10-year's experience. Kaohsiung J Med Sci 2002; 18:221-8. (level 3b)

5. Csendes A, Burdiles P, Maluenda F, Diaz J, Csendes P, Mitru N. Simultaneous bacteriologic assessment of bile from gallbladder and common bile duct in control subjects and patients with gallstones and common duct stones. Arch Surg 1996;131:389-94. (level 2b)

6. Csendes A, Mitru N, Maluenda F, Diaz J, Burdiles P, Csendes P, et al. Counts of bacteria and pyocites of choledochal bile in controls and in patients with gallstones or common bile duct stones with or without acute cholangitis. Hepatogastroenterology 1996; 43:800-6. (level 2b)

7. Brismar B, Jalakas K, Malmborg A, Strandberg A. The significance of bacteriological findings at cholecystectomy. Acta Chir Scand 1986;530(Suppl):35-8. (level 3b)

8. Jarvinen H. Biliary bacteremia at various stages of acute cholecystitis. Acta Chir Scand 1980;146:427-30. (level 2b)

9. Hanau L, Steigbigel N. Acute (ascending) cholangitis. Infect Dis Clin North Am 2000;14:521-46. (level 4)

10. Westphal J, Brogard J. Biliary tract infections: a guide to drug treatment. Drugs 1999;57:81-91. (level 5)

11. Sinanan M. Acute cholangitis. Infect Dis Clin North Am 1992;6:571-99. (level 5)

12. Marne C, Pallares R, Martin R, Sitges-Serra A. Gangrenous cholecystitis and acute cholangitis associated with anaerobic bacteria in bile. Eur J Clin Microbiol 1986;5:35-9. (level 3b)

13. Claesson B, Holmlund D, Matzsch T. Microflora of the gallbladder related to duration of acute cholecystitis. Surg Gynecol Obstet 1986;162:531-5. (level 2b)
14. Nielsen M, Justesen T. Anaerobic and aerobic bacteriological studies in biliary tract disease. Scand J Gastroenterol 1976;11:43746. (level 3b)

15. Hanau L, Steigbigel N. Cholangitis: pathogenesis, diagnosis, and treatment. Curr Clin Top Infect Dis 1995;15:153-78. (level 4)

16. Thompson JE Jr, Bennion R, Pitt HA. An analysis of infectious failures in acute cholangitis. HPB Surg 1994;8:139-45. (level 4)

17. Dellinger RP, Carlet JM, Masur H, Gerlach H, Calandra T, Cohen $\mathrm{J}$, et al. for the Surviving Sepsis Campaign Management Guidelines Committee. Surviving Sepsis Campaign guidelines for management of severe sepsis and septic shock. Crit Care Med 2004;32:858-73. (level 4)

18. Gilbert D, Moellering R Jr, Sande M. The Sanford guide to antimicrobial therapy. 33rd ed. Hyde Park, VT: Antimicrobial therapy; 2003. (level 4)

19. Brunton LL, Lazo JS, Parker KL, editors. Goodman and Gilman's the pharmacological basis of therapeutics. 11th ed. New York: McGraw-Hill; 2006. (level 4)

20. Leung J, Ling T, Chan R, Cheung S, Lai C, Sung J, et al. Antibiotics, biliary sepsis, and bile duct stones. Gastrointest Endosc 1994; 40:716-21. (level 3b)

21. Martinez O, Levi J, Devlin R. Biliary excretion of aztreonam in patients with biliary tract disease. Antimicrob Agents Chemother 1984;25:358-61. (level 4)

22. Leung J, Chan C, Lai C, Ko T, Cheng A, French G. Effect of biliary obstruction on the hepatic excretion of imipenem-cilastatin. Antimicrob Agents Chemother 1992;36:2057-60. (level 3b)

23. Leung J, Chan R, Cheung S, Sung J, Chung S, French G. The effect of obstruction on the biliary excretion of cefoperazone and ceftazidime. J Antimicrob Chemother 1990;25:399-406. (level 4)

24. Blenkharn J, Habib N, Mok D, John L, McPherson G, Gibson R, et al. Decreased biliary excretion of piperacillin after percutaneous relief of extrahepatic obstructive jaundice. Antimicrob Agents Chemother 1985;28:778-80. (level 4)

25. van den Hazel S, de Vries X, Speelman P, Dankert J, Tytgat G, Huibregtse K, et al. Biliary excretion of ciprofloxacin and piperacillin in the obstructed biliary tract. Antimicrob Agents Chemother 1996;40:2658-60. (level 4)

26. Takada T, Hanyu F, Fukushima Y, Uchida Y, Imaizumi T, Yasuda $\mathrm{H}$. Excretion of antibiotics into human bile juice in patients with obstructive jaundice (in Japanese). Jpn J Gastroenterol 1976;73:39_ 47. (level 4)

27. Levi J, Martinez O, Malinin T, Zeppa R, Livingstone A, Hutson D, et al. Decreased biliary excretion of cefamandole after percutaneous biliary decompression in patients with total common bile duct obstruction. Antimicrob Agents Chemother 1984;26:944-6. (level 4)

28. Boey J, Way L. Acute cholangitis. Ann Surg 1980;191:264-70. (level 5)

29. Thompson JE Jr, Tompkins R, Longmire WJ. Factors in management of acute cholangitis. Ann Surg 1982;195:137-45. (level 4)

30. Muller E, Pitt HA, Thompson JE Jr, Doty J, Mann L, Manchester B. Antibiotics in infections of the biliary tract. Surg Gynecol Obstet 1987;165:285-92. (level 2b)

31. Gerecht W, Henry N, Hoffman W, Muller S, LaRusso N, Rosenblatt J, et al. Prospective randomized comparison of mezlocillin therapy alone with combined ampicillin and gentamicin therapy for patients with cholangitis. Arch Intern Med 1989;149:1279-84. (level 2b)

32. Thompson JE Jr, Pitt HA, Doty J, Coleman J, Irving C. Broad spectrum penicillin as an adequate therapy for acute cholangitis. Surg Gynecol Obstet 1990;171:275-82. (level 2b)

33. Chacon J, Criscuolo P, Kobata C, Ferraro J, Saad S, Reis C. Prospective randomized comparison of pefloxacin and ampicillin plus gentamicin in the treatment of bacteriologically proven biliary tract infections. J Antimicrob Chemother 1990;26(Suppl B):16772. (level 2b) 
34. Thompson JE Jr, Bennion R, Roettger R, Lally K, Hopkins J, Wilson SE. Cefepime for infections of the biliary tract. Surg Gynecol Obstet 1993;177(Suppl):30-4. (level 2b)

35. Sung J, Lyon D, Suen R, Chung S, Co A, Cheng A, et al. Intravenous ciprofloxacin as treatment for patients with acute suppurative cholangitis: a randomized, controlled clinical trial. J Antimicrob Chemother 1995;35:855-64. (level 2b)

36. Solomkin J, Mazuski J, Baron E, Sawyer R, Nathens A, DiPiro $\mathrm{J}$, et al. Guidelines for the selection of anti-infective agents for complicated intra-abdominal infections. Clin Infect Dis 2003;37: 997-1005. (level 4)

37. Investigators of the Piperacillin/Tazobactam Intra-abdominal Infection Study Group. Results of the North American trial of piperacillin/tazobactam compared with clindamycin and gentamicin in the treatment of severe intra-abdominal infections. Eur J Surg 1994;573(Suppl):61-6. (level 2b)

38. Sales J, Sutcliffe M, O'Grady F. Excretion of clindamycin in the bile of patients with biliary tract disease. Chemotherapy 1973;19: 11-5. (level 4)

39. Csendes A, Becerra M, Burdiles P, Demian I, Bancalari K, Csendes P. Bacteriological studies of bile from the gallbladder in patients with carcinoma of the gallbladder, cholelithiasis, common bile duct stones and no gallstones disease. Eur J Surg 1994;160:363-7. (level 2b)

40. Csendes A, Fernandez M, Uribe P. Bacteriology of the gallbladder bile in normal subjects. Am J Surg 1975;129:629-31. (level 3b)

41. Kune G, Schutz E. Bacteria in the billary tract. A study of their frequency and type. Med J Aust 1974;1:255-8. (level 3b)

42. Orda R, Berger S, Levy Y, Shnaker A, Gorea A. Penetration of ceftriaxone and cefoperazone into bile and gallbladder tissue in patients with acute cholecystitis. Dig Dis Sci 1992;37:1691-3. (level 4)

\section{Discussion at the Tokyo International Consensus Meeting}

\section{The issue of the Significant difference between the United States and Japan in antimicrobial dosing regimens}

Harumi Gomi (Japan): In the United States, ampicillin/ sulbactam - one of the most commonly used agents for intraabdominal infections - the regular dosage for adult patients with normal renal function is $3 \mathrm{~g}$ intravenously every 6 hours, and the total dosage is $12 \mathrm{~g}$ per day. On the other hand, in Japan, the legally approved dosage is $3 \mathrm{~g}$ intravenously twice a day, meaning the maximum daily dose is $6 \mathrm{~g}$. Another example is piperacillin/tazobactam. The FDA-approved dosage is 3.37$4.5 \mathrm{~g}$ intravenously every $6-8 \mathrm{~h}$, meaning $13.5-17.5 \mathrm{~g}$ per day. On the other hand, in Japan, the regular dose or legally approved dose is $2.5 \mathrm{~g}$ intravenously twice a day, meaning $5 \mathrm{~g}$ per day is the maximum. [In regard to] aminoglycosides: [for] gentamicin; in the United States, the regular dosage is $1-1.7 \mathrm{mg}$ per kg every $8 \mathrm{~h}$, or $4.5-5.0 \mathrm{mg}$ per $\mathrm{kg}$ every $24 \mathrm{~h}$ as a once-daily dosage. Therefore for adult patients with a body weight of up to $50 \mathrm{~kg}$, the daily dose is $225-250 \mathrm{mg}$. But again, in Japan, the maximum dose is $80-100 \mathrm{mg}$ per day, regardless of body weight. So there is a significant issue and difference.

\section{How long should antimicrobial agents be given for} patients with acute cholangitis?

Joseph S. Solomkin (USA): The other point I will make, just to relay our experience in North America, is that there is increasing emphasis on shortened duration of therapy, and typically now the standard recommendation for treatment would be approximately 7-10 days until the patient is afebrile, has resolved their infection clinically, and is taking oral intake. There are a lot of people who think that that is too long; that in fact 5 days may be the optimal therapy, so I think that is another very important area to look at, because certainly the longer patients are on these very broadspectrum agents, the greater the potential harm in terms of superinfection and toxicity.

Henry A. Pitt (USA): That was my point as well. I give a short course if there is no bacteremia, and then try to stop quickly, but I give a real course of 7-10 days if there is bacteremia.

Joseph Solomkin: Has anybody ... I would just like to ask one question since I think you people have more experience than I do with this; if a patient has an episode of cholangitis, is short-course treatment - say 5 days - is there a risk they will develop liver abscesses? So when we are talking about the duration of therapy, should that be a factor in it?

Henry Pitt: I think it depends a lot on the exact clinical situation. I mean, we see cholangitis most often now in patients who have indwelling stents, who come in and they get their stent changed, and then the bile is flowing again and the cholangitis goes away quickly, and they either have had a liver abscess or not when they come in, and you figure that out, if they do not respond to the usual therapy and/or they have blood cultures.

Serafin C. Hilvano (Philippines): I would also agree that we set a minimum number of days for the therapy.

Thomas R. Gadacz (USA): There are a lot of specifics that have been brought up, such as liver abscess, you would treat a patient for a long period of time. Patients where the acute cholangitis may be simply be due to a plugged-up stent which gets changed very quickly, in which case short-term therapy would be probably very appropriate. So I think that the absolute determination here is not one that that is trying to be a solution, but really a guideline and that is stated in the question, "should be." The specific situation then could be altered depending upon what the exact condition is. 


\section{Should biliary penetration be considered important in the selection of therapeutic antimicrobials in acute cholangitis?}

Henry Pitt: The first point has to do with biliary penetration. I think that there is a spectrum of disease, and initially before drainage the biliary penetration probably makes no difference, and having good blood levels is very important. But I think after drainage, I imagine, although there are no good data, that their biliary penetration gradually goes up and that there may be some advantage 3 or 4 days into an illness when someone is very sick, I do not know.

Chen-Guo Ker (Taiwan): In cases of obstruction, the penetration of antibiotics was very low, in the studies more than 10 years ago. So it is better to give the drainage in the first acute phase. But during the acute phase, we have to keep the antibiotics for prevention of the systemic bacteremia; so that you do not mention. It is not necessary to care about the penetration into the bile. But another thing which is very important; antibiotic penetration into the bile, this should be combined with the ligand-specific protein. So in cases of patient with low albuminemia, last penetrated into the bile must be very low. So we have to care about the timing of the giving of antibiotics and what kind of antibiotics we use. It is my opinion. Thank you.

(Voting was done)

Joseph Solomkin: You know, I think the numbers, particularly from our Japanese hosts, are strong enough so that in the guidelines we should say or make the statement that it is the opinion of the Japanese that biliary penetration is important.

Steven M. Strasberg (USA): But is the other point not given that what we are here to do is that there is not good evidence from the literature of the importance of this factor?

Atsushi Tanaka (Japan): Well, as I have said, there is very little evidence suggesting the importance of this.

Steven Strasberg: Well, that is what I mean; there is very little evidence, so it is really a point that we cannot make a rational decision about it, so it is about as authoritarian as you can get.

Joseph Solomkin: That is why it was brought up for discussion, but I think here that Dr. Tanaka made the point very clearly that that was the case; that the superiority just is not there, it has not been demonstrated.
Conversely, if you have a group of practitioners who strongly believe something that is not critical to the health of the patient, I would be more concerned of risking their not using the guidelines at all. That is a very big question.

Yoshifumi Kawarada (Japan): Sir. I have to ask Dr. Gomi, what do you think about the biliary penetration by antibiotics in acute cholecystitis?

Harumi Gomi: Well, since all my training was done in the United States, I am more towards the United States position. This means that I do not consider the penetration of the biliary tract.

Yoshifumi Kawarada: Yes, I had the same opinion. I had a bias. I was educated in the United States, always being against the penetration, it is not so important; but for Japanese people, 71\% say "Yes."

Steven Strasberg: I find it very difficult to understand how we can publish a guideline that says anything that is not a reflection of the best available evidence; and think that whether someone is going to follow a guideline or not is a second degree of relevance, or a second degree of what we should be considering. I do not know this literature, but if the literature says that drugs that do penetrate the biliary epithelium do not do any better than drugs that do not penetrate the biliary epithelium, then just as you have said before, the evidence is that it is a factor of no importance or minor importance, and I think the guidelines should say that.

Joseph Solomkin: The reservation - I appreciate you saying that - the reservation I have is that these are consensus guidelines, so that they are guidelines that basically ... these guidelines, as far as I am concerned, or were I to write them would say, "The evidence is such and such; at the consensus meeting, nonetheless, the panelists believe because of current common practice, that such and such is okay." I think you have to do both things; state the facts and then I do not think you can discredit the consensus.

Henry Pitt: Part of the problem is that we have no good evidence. The paper that is quoted as the best evidence is Michael Keith-Floyd's paper that was published in 1974, and it was a retrospective analysis of whether people were treated with gentamicin or not. That is not good evidence either. So we have to make a recommendation, and then we say it is based on A-, B-, C-, D-, or E-level evidence, and this will be a lowerlevel evidence recommendation. 